\title{
Variables asociadas al uso, socialización y riesgo de los jóvenes frente a los dispositivos portátiles inteligentes con conectividad a Internet. El caso de la educación secundaria pública mexicana
}

Variables associated with the use, socialization and risk of young people facing portable devices with Internet connectivity. The case of Mexican public secondary education

\author{
Gustavo Adolfo LEÓN DUARTE \\ Universidad de Sonora - México \\ gustavoadolfoleon@gmail.com \\ Dora Yéssica CAUDILLO RUIZ \\ Universidad de Sonora - México \\ doracaudilloruiz@gmail.com \\ Carlos René CONTRERAS CÁZAREZ \\ Universidad de Sonora - México \\ renecazarez@gmail.com
}

\begin{abstract}
Cómo citar este artículo: LeÓn DuARTE, Gustavo Adolfo, CAUDILlo Ruiz, Dora Yéssica y ConTRERAS CÁZAREZ, Carlos René (2015): «Variables asociadas al uso, socialización y riesgo de los jóvenes frente a los dispositivos portátiles inteligentes con conectividad a Internet. El caso de la educación secundaria pública mexicana», Mediaciones Sociales, №14, pp. 81-101. DOI: http://dx.doi.org/10.5209/rev_MESO.2015.n14.51560
\end{abstract}

Recibido: 14 de noviembre de 2014.

Aceptado: 13 de mayo de 2015.

\section{RESUMEN}

En el artículo se discuten los hallazgos del tercer diagnóstico anual consecutivo que entre 2012 y 2014 mide las variables asociadas al uso, consumo y socialización que presentan los jóvenes estudiantes de secundarias públicas de la Ciudad de Hermosillo, Sonora (México) frente a las tecnolo- 
gías digitales y a los dispositivos portátiles con conexión a Internet. El estudio busca también identificar las variables asociadas al riesgo, su reacción ante el mismo y los hábitos o medidas de seguridad que adoptan los jóvenes o sus padres y profesores en la utilización de dichas tecnologías. Se trata de una investigación con financiamiento externo. Utiliza el multiplismo crítico como perspectiva teórica interdisciplinar y estrategias metodológicas que devienen de un modelo mixto integrado que permite el análisis de datos de manera transversal y temporal. Contempla dos fases metodológicas y una combinación de técnicas cuantitativas y cualitativas. El análisis cuantitativo examinó la aplicación de 2.907 cuestionarios. La fase cualitativa evalúa la implementación de siete grupos focales.

Palabras ClaVe: dispositivos móviles, educación, internet, jóvenes, México.

\section{ABSTRACT}

The article presents the findings of the diagnostic between 2012 and 2014 about uses, attitudes, habits, skills and behaviors that present the young students of public secondary schools in the city of Hermosillo, Sonora (Mexico) with respect to technologies digital and portable device with Internet access. The study also discusses the results regarding the risks, their reaction to them and the habits or adopt security measures that youth or their parents and teachers in the use of these technologies. It is a research with externally funded. Use the critical multiplism as interdisciplinary theoretical perspective and methodological strategies that become an integrated mixed model for the analysis of cross-sectional data temporarily. Methodology involves two stages and a combination of quantitative and qualitative techniques. The quantitative analysis examined the application of 2907 questionnaires. The qualitative phase evaluated the implementation of seven focus groups.

KEYWORDS: mobile devices, education, internet, youth, Mexico.

Sumario: 1. Introducción. 2. Fundamentación teórica. 3. Diseño metodológico. 4. Resultados del estudio. 4.1. Usos, consumos y preferencias en Internet. 4.2. Socialización. Interacción comunicativa. 4.3. Regulación, mediación y control de padres y profesores. 5. Conclusiones. Variables asociadas. Bibliografía.

\section{INTRODUCCIÓN}

En los últimos años, las investigaciones realizadas sobre el uso y consumo de las tecnologías digitales por parte de las y los menores de edad han mostrado una evidente preocupación por el uso de los distintos dispositivos portátiles con conexión a Internet y su incidencia en los procesos de formación educativa. Los diagnósticos muestran una tendencia global de incremento tanto en el tiempo de uso como en las preferencias de contenido, la accesibilidad, conectividad y el acceso a dispositivos tecnológicos inteligentes móviles. En el caso de México, la preocupación se incrementa cuando se comprueba la recurrencia de uso a más temprana edad. Diversos estudios han aportado información que permite lograr un diagnóstico sobre el papel fundamental, casi imprescindible, de las tecnologías digitales en la vida de los jóvenes. 
Esto lo convierte en una problemática en consecuente cambio donde la constante es la inmediatez y la obsolescencia. Frente a este panorama se construye un nuevo reto para los sistemas educativos: reducir la brecha digital generacional que ha incidido directamente en el proceso de enseñanza aprendizaje. A esto, se le suma las situaciones de riesgo a las que se ven expuestos los menores. No pocas situaciones de riesgo han derivado en una serie de problemas para los jóvenes y encuentran resolución sólo desde distintas instancias de procuración de justicia, incluyendo los procedimientos penales.

Diversas investigaciones internacionales coinciden en buscar estrategias que apunten hacia una navegación segura de los menores. Instan a promover el uso responsable de las Tecnologías de Información y Comunicación (TIC) a través de la alfabetización digital, tanto de los menores, padres de familia y profesores. Se hace más evidente la urgente necesidad de ver y entender el avance tecnológico como un vehículo idóneo para el desarrollo de las capacidades de razonamiento, creatividad y comunicación. También, de (re)plantearse cuestiones básicas de entendimiento partiendo del cómo y de qué forma los menores de edad están integrando los dispositivos portátiles inteligentes y qué contenidos, aplicaciones y relaciones moldean dicha integración. Así mismo, buscan conocer qué implicaciones puede tener a la hora de diseñar el objeto y la función de la escuela en la sociedad actual. Son muchas las evidencias que en el plano internacional existente sobre las ventajas que generan las tecnologías y el Internet en el aula. Por ejemplo, desde el punto de vista del profesorado estadounidense del nivel secundaria se considera que las tecnologías digitales se han convertido en el centro de su enseñanza y la profesionalización. Al mismo tiempo, que Internet, los teléfonos móviles y las redes sociales han traído consigo nuevos desafíos y un impacto global en la enseñanza y su trabajo directo en el aula: a) Para un $92 \%$ de los profesores estadounidenses de secundaria Internet tiene un "impacto importante" en su capacidad para acceder a los contenidos, recursos y materiales para su enseñanza; b) Para un 69\% del colectivo el Internet tiene un "impacto importante" en su capacidad de compartir ideas con otros profesores; y c) Para un $67 \%$ el Internet tiene un "impacto importante" en su capacidad de interactuar con los padres y mejorar su interacción con los estudiantes (Purcel, Heaps, Buchanan y Friedrich, 2013).

En el caso de poblaciones urbanas, es frecuente que el joven de secundaria en México viva en hogares con un reducido número de miembros. Con la madre incorporada plenamente al mercado laboral y con una evidente dificultad para conciliar la vida laboral, personal y familiar. Además, la creciente inseguridad de una década a esta parte en ciertas regiones de México ha dificultado que los menores puedan ocupar parte de su tiempo libre jugando al aire libre. 
La confluencia de todos estos factores en el caso de los jóvenes mexicanos explica por qué muchos han crecido rodeados de todo tipo de dispositivos tecnológicos portátiles. Esta Generación Interactiva Mexicana (GIM), caracterizada por el alto grado de posesión de pantallas y tecnologías digitales, le han añadido un mayor grado de interactividad entre el hombre y la tecnología, o entre los propios seres humanos gracias a ella. Esto les confiere unas características algo peculiares que conviene conocer así sea de manera general y rápida:

1) Una primera característica de la GIM es la gran facilidad para enviar, recibir y procesar información velozmente. La información es adquirida por variados canales. La selección tanto de la información como del canal responde a un impulso muy rápido que no siempre ha sido pensado ni implica necesariamente una posterior reflexión. Por ello, de acuerdo con estudios previos, podemos afirmar que por lo general el joven de secundaria en México no realiza un análisis crítico de la información recibida sino más bien al contrario. Necesitan saber para qué les sirve lo que van a realizar en el mismo instante en el que se proponen realizarlo. Por lo general la revisión crítica de la información no va más allá del mensaje que le produce la imagen. Sólo si no ha satisfecho su voluntad de saber a través de esta recurrirá al texto. Ciertamente, tienen la inteligencia visual muy desarrollada. Se mueven en un medio en el que la información se organiza de una manera absolutamente distinta a la utilizada en la escritura convencional. Por ello, los libros "en papel" les pueden resultar extraños y les cuesta tanto la lectura pausada de un texto.

2) La continua interactividad con dispositivos inteligentes puede llegar a las 24 horas. Su competencia tecnológica se basa en la primacía de hacer antes que hacer bien, por lo que al joven le cuesta mucho reflexionar sobre sus propias actitudes y conductas (León, Castillo, Montes y Caudillo, 2013). Además, necesitan recibir una recompensa inmediata en lugar de gratificaciones que se materializarán a medio o largo plazo.

3) Tienen la atención más diversificada. Por eso son capaces de realizar varias tareas de forma simultánea como, por ejemplo: escuchar música mientras estudian a la vez que sostienen conversaciones en varias redes sociales. Se debe enfatizar que el texto escrito les sirve para ilustrar la imagen: sólo en el caso de que no comprendieran ésta se acudiría a aquel. No suelen pensar ni evaluar la validez o bondad de la respuesta obtenida.

Ahora, las y los hijos aplican y conocen más la tecnología y la aplicación de dispositivos tecnológicos que sus padres y madres. Algo que, como hemos visto, está resultando crucial a la hora de configurar su "forma de ser", sus valores y actitudes (León, Castillo, Montes y Caudillo, 2013). Los niños y niñas declaran ser los "expertos tecno- 
lógicos" del hogar. Han aprendido a manejar la tecnología digital de manera autónoma, sin la mediación educativa de un adulto, reconocen ser los que más saben sobre ella y los que hacen un uso más intensivo y multifuncional. Por tanto, es evidente que frente a las tecnologías digitales y a Internet, la generación interactiva va por delante en conocimiento y uso, hecho que sitúa a profesores y progenitores en clara desventaja. Por lo mismo, hoy el menor de edad puede llegar a cuestionar la autoridad de estos para ejercer cualquier mediación y control sobre dichos dispositivos.

Por lo anterior, esta investigación parte de la hipótesis de que el uso, consumo y producción interactiva de contenidos digitales mediante dispositivos portátiles con conectividad a Internet sitúa al joven de secundaria en condiciones de inseguridad. Esta condición está directamente asociada a varios factores donde se destaca: 1) EI dominio que tengan padres y profesores en el uso de los dispositivos inteligentes y 2) Al conocimiento y sentido de prevención de riesgo para diseñar y aplicar estrategias de regulación en el uso seguro de Internet. La magnitud y orientación de sus efectos dependerá de las preferencias, los hábitos, la socialización y las competencias tecnológicas personales que el joven construya en el mundo de la vida cotidiana y las redes sociales.

Es por ello que el estudio centra su atención en identificar las variables asociadas con el uso, socialización y riesgos de los jóvenes estudiantes de secundarias públicas del Municipio de Hermosillo, Sonora (México) al utilizar dispositivos portátiles con conectividad a Internet. Hay que señalar que este proyecto persigue dos objetivos diferentes que requieren estrategias de muestreo distintas: el primer objetivo es poder estimar la prevalencia de diferentes variables relacionadas con el uso y consumo de diversos dispositivos móviles con conexión a Internet en jóvenes de secundaria. Segundo, lograr una representatividad muestral precisamente mediante el muestreo aleatorio simple y a partir de una lista de unidades de muestreo del cual se pueden elegir aleatoriamente los sujetos de interés. En el caso del presente estudio el universo se fundamenta en jóvenes estudiantes de secundaria de entre 12 y 15 años de edad e inscritos en alguno de los 23 centros de educación secundaria del Municipio de Hermosillo, Sonora, México.

\section{FUNDAMENTACIÓN TEÓRICA}

Cuando nos acercamos al estudio de Internet y de las nuevas tecnologías en general, podemos adoptar varios puntos de vista sobre el fenómeno, diferentes posiciones que permitan acercarnos a su comprensión. En este apartado se presenta el modelo teórico el cual deviene de una perspectiva teórica interdisciplinar específica. Partimos de integrar al debate la necesidad de contemplar lo disciplinar y lo interdisciplinar desde diferentes dimensiones que permitan matizar las posibilidades reales de anali- 
zar y explicar un fenómeno social complejo y multidimensional. Entendemos en este sentido que un abordaje focalizado desde una disciplina resulta incompleto. Una problemática social compleja y de carácter multidimensional como nuestro objeto, temática y problemática social abordada no puede ser trabajada si no se toma en cuenta, por ejemplo, variables psicológicas, sociológicas y culturales. Esto obviamente exige algún grado de diálogo entre distintas disciplinas.

En el contexto del papel de las TIC como elemento nuclear de la globalización, Repko (1998) y Patry (2013) afirman que la interdisciplinariedad debe verse como un proceso que permite solucionar conflictos, comunicarse, cotejar y evaluar aportaciones disciplinares a la vez que es sugerente la integración de datos sobre marcos epistemológicos integradores previos. Desde una posición amplia, la interdisciplinariedad puede entenderse como un proceso y una filosofía organizacional aplicada a la investigación académica científica. Es, por tanto, una forma de pensar y de proceder para enfrentar al conocimiento de la complejidad de la realidad y resolver cualquiera de los complejos problemas que se plantea y se hacen evidentes, tanto al interior de la estructura de cada disciplina como en sus prácticas científicas, tratadas aquí como un proceso histórico de conocimiento y de reconocimiento de sus agentes e instituciones (León, 2014: 15).

Las problemáticas urbanas obtienen cada vez más atención conforme a la expansión de la población mundial que vive en las ciudades. Recientemente ha habido un aumento significativo en la investigación sobre las problemáticas sociales. Las ciencias sociales están comenzando a vincularse cada vez más con la antropología, la comunicación, la psicología y otros campos. Por ejemplo, en los últimos años los estudios de innovación tecnológica parecen ganar la atención no sólo en estudios de ciencia y tecnología, que han sido campos relativamente segmentados y separados de las principales disciplinas en ciencias sociales, sino también en estudios de economía, sociología y antropología, entre otras (Craig y Tracy, 2014; Craig, 1999). Se debe reconocer que la tendencia interdisciplinar, multi y transdisciplinar, ha existido desde el mismo momento en que surgieron las disciplinas. Éstas han constituido a veces el origen de nuevas disciplinas, incluyendo algunas que no se cristalizaron y que finalmente desaparecieron. Esta dinámica de cooperación interna y de fertilización cruzada entre disciplinas no sólo existe entre las ciencias sociales. También es un elemento de las interacciones entre las ciencias sociales y otros campos del conocimiento, especialmente en las humanidades y las ciencias naturales (Silbereisen, Ritchie y Overmier, 2010). Por otro lado, Shadish (1993) mediante la iniciativa del Multiplismo Crítico (MC) sugiere el uso de múltiples teorías, hipótesis, métodos, investigadores, disciplinas y la síntesis de conocimiento, en un intento por explicar la realidad. Expresa que la combinación de múltiples estrategias y visiones elimina el sesgo que presenta cada una de ellas por separado y elimina también el favoritismo intelectual por las ideas 
propias. En todo caso, el cuestionamiento que aquí nos planteamos es si se puede aplicar la aproximación del multiplismo crítico en la práctica de la investigación interdisciplinar de la generación interactiva. Esto puede también llevar a considerar la explicación del todo desde una perspectiva única. Ese riesgo se reduce si el enfoque de la investigación es multiplista, ya que la ciencia puede considerarse como única, pero con múltiples aristas.

En la figura 1 se expresa el tratamiento de los usos, la socialización y el riesgo en Internet y sus respectivos modelos (restringidos) disciplinares anidados. Este modelo inclusivo, con el cual hemos trabajado en el estudio 2014 no sólo contempla las relaciones particulares que se estudian al interior de cada disciplina, sino también las interacciones entre objetos y las aportaciones específicas disciplinares de diferentes campos. En este caso, es posible efectuar contrastes entre los modelos disciplinarios, estimando su poder explicativo particular y probando el poder explicativo del modelo inclusivo interdisciplinario. Las estrategias de elaboración e implementación del modelo sigue la propuesta del MC al contemplar los modelos anidados a la postulación de modelos interdisciplinarios. También, en este estudio hemos agregado la revisión de teorías y la integración de las mismas. Incluye, como ya adelantábamos, un diseño metodológico de corte mixto y utilizando una hipótesis de investigación basada en aproximaciones teóricas y campos disciplinares de estudio que comparten, por lo menos, uno o más elementos en común. Adicionalmente fue conveniente contar con aproximaciones teóricas que, de inicio, consideraban la integración y el contraste como sus constituyentes básicos, dando, por lo tanto, cabida a la conmensurabilidad de los modelos.

Figura 1. Modelos disciplinares anidados en un modelo inclusivo interdisciplinario aplicado a la GIM

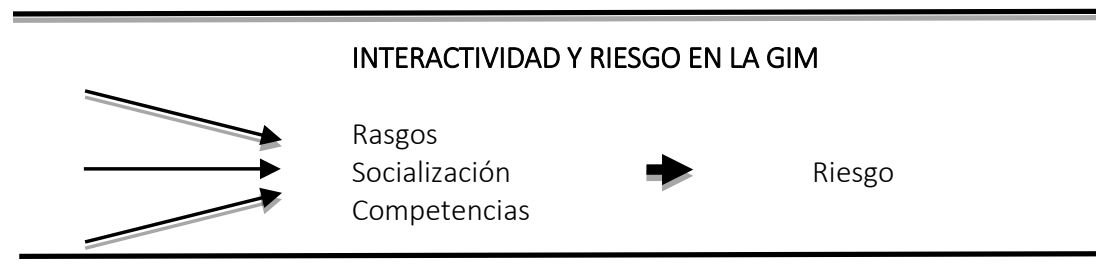
1) Psicología:
Usos, Consumos y Rasgos

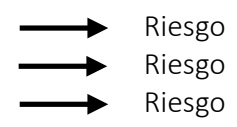
2) Sociología:
3) Educación:
Socialización (en línea)
Competencias
Riesgo

Las variables asociadas a identificar los cambios que se están perfilando en los usos y hábitos online de los adolescentes es uno de los propósitos de la presente investigación. Particularmente debido a la fuerte irrupción de dispositivos portátiles 
con conexión a Internet. Como ya se indicó anteriormente, esto ha incidido directamente en los hábitos de consumo, en las preferencias de los jóvenes, así como en las implicaciones socioculturales de estos procesos. Entre las principales variables se destaca aquí el uso en tiempo y los hábitos en actividades que los usuarios utilizan muy frecuentemente, con especial incidencia en aquellas actividades que les permiten mantener el contacto (socialización) y compartir contenidos con sus pares así como actividades de ocio y entretenimiento. Las variables de usos y hábitos tratados en esta investigación, serán presentadas con especial vinculación a los trabajos de Sonia Livingstone $(2002,2007,2008,2010,2014)$, en el marco del proyecto multinacional europeo sobre riesgos y seguridad de niños europeos. Dichos estudios se han centrado tanto en los usos y experiencias de los menores en Internet como en los riesgos a los que se pueden enfrentar como son el cyberbulling, la pornografía, o la invasión de la privacidad, así como en aspectos como los niveles de alfabetización y competencia digital. Durante el proceso de apropiación de las TIC se presentan varios factores determinantes para poder llevar a cabo dicho proceso. Una vez que se tiene acceso físico a dispositivos de comunicación, generalmente por posibilidad económi$\mathrm{ca}$, se ha roto la principal barrera de acceso. Una vez logrado esto, el individuo se mantiene en constante exposición al uso del dispositivo, la necesidad de mantenerse conectado lo obliga a "aprender" a utilizarlo. A este proceso le llamaremos tecnologización del individuo. Posteriormente se adquiere preferencias de uso y contenido lo cual genera un hábito de uso. Es así como el objetivo de "usar" un dispositivo se relaciona a la necesidad de utilizarlo como herramienta de comunicación. Cuando el uso se hace constante y frecuente, el individuo comienza a generar hábitos que están relacionados con patrones y conductas. Por ejemplo, Livingstone clasifica las formas de asimilación por parte de los usuarios, así como su grado de comprensión de los mensajes, la capacidad de evaluación, las posiciones individuales ante el medio, el alcance crítico, etc. Sus estudios advierten de los distintos comportamientos en función de variables socio-demográficas (edad, sexo, formación, clase social) y otras de naturaleza psicológica. La dimensión semiológica del acercamiento se combina con los trazos psico-sociológicos, de modo que las pautas constructivas de la representación simbólica aparecen relacionadas con perfiles humanos y grupales concretos (Livingston, 2008). Otros estudios se preocupan, entre otros aspectos, por el uso intensivo (Lenhart, Madden y Smith, 2008; Lenhart, Purcell, Smith y Zickuhr, 2010) o de la creciente influencia de determinados dispositivos como pueden ser los celulares (Purcell, 2011). A su vez, existen estudios que acentúan el estudio de los usos a partir de temas educativos y la alfabetización (Eynon y Helsper, 2014), o a partir de la capacidad multitarea de las nuevas generaciones (Bowman, Waite y Levine, 2014), las relaciones e influencia del contexto familiar (Duerager y Livingstone, 2012), los aspec- 
tos referidos a las diferencias de género (Valkenburg, Peter y Schouten, 2006), el impacto de las diferencias offline o la creación de contenidos (Buckingham, 2013).

La socialización también conocida como internalización es tratada aquí como un proceso mediante el cual el individuo adopta los elementos socioculturales de su medio y los integra a su personalidad para adaptarse a la sociedad en la que vive. Dicho en otros términos, socializar es el proceso que afecta a diversos aspectos del desarrollo sobre todo en la niñez y adolescencia, y que abarcan el crecimiento, cambios psicológicos y emocionales y la integración social. Cuando hablamos de socialización, es necesario referirnos al proceso de socialización misma que no sólo plantea el aprendizaje cognoscitivo, sino también el consentimiento de los sujetos.

Así el proceso de la socialización primaria comporta algo más que un aprendizaje puramente cognoscitivo. Se efectúa en condiciones de enorme carga emocional. Existen, ciertamente, buenos motivos para creer que sin esa adhesión emocional a otros significantes, el proceso de aprendizaje sería difícil, cuando no imposible. El niño se identifica con los otros significantes en una variedad de formas emocionales; pero sean éstas cuales fueren, la internalización se produce sólo cuando se produce la identificación. El niño acepta los roles y actitudes de los otros significantes, o sea, los internaliza y se apropia de ellos. Por esta identificación con los otros significantes, el niño se vuelve capaz de identificarse él mismo, de adquirir una identidad subjetivamente coherente y plausible.

Podemos afirmar que, dependiendo de la etapa de vida de los individuos, la aceptación del bagaje cultural se lleva a cabo de manera diferente. Durante la niñez y los primeros años de la adolescencia, la socialización se realiza por lo general al interior de grupos afectivos, culturalmente homogéneos, como la familia, la iglesia, los amigos. Siguiendo los estudios de Berger y Luckmann $(1968,1997)$, entenderemos aquí por socialización primaria la que acontece fundamentalmente durante la infancia. Después se produce lo que Berger y Luckmann denominan socialización secundaria. Es decir, aquella que tiene lugar sobre todo en la adolescencia y primera juventud, aunque prosiga a todo lo largo de la existencia. La socialización secundaria consistiría en la tarea de iniciarse en los roles propios de la vida en sociedad: los relacionados con la división del trabajo, la diversidad profesional, participación social, grupos de diversión, religiosos, como también el rol sexual y las relaciones intersexuales (Berger y Luckmann, 1968).

En el mismo sentido, Berger y Luckmann; (1997) expresan que la acción de otras personas respecto del niño está determinada en gran medida por esquemas de experiencia y actos extraídos del depósito social de sentidos. "El niño aprende, de una manera progresiva, a comprender y a entender el sentido de las acciones de sus contrapartes (...) así, durante el proceso el niño desarrolla progresivamente su identidad 
personal" (Berger y Luckmann, 1997: 44). Una vez que el joven llega a comprender el sentido de sus acciones, también entiende que a él se le considera en principio responsable de sus propias acciones. "Esto es lo que constituye la esencia de la identidad personal: el control subjetivo de la acción de la que uno es objetivamente responsable" (Berger y Luckmann, 1997: 44-45).

$\mathrm{Si}$ atendemos a las investigaciones que se han venido realizando en los últimos años en torno a los posibles riesgos asociados al uso que jóvenes y adolescentes hacen de las nuevas tecnologías, encontramos entre las temáticas tratadas con mayor frecuencia: contenidos inapropiados, contactos con desconocidos, amenazas a la privacidad y, en menor medida, riesgos relacionados con el comercio electrónico (Staksrud, Ólafsson y Livingstone, 2013). Por otro lado, el crecimiento de la sociedad de la información viene acompañado por nuevas amenazas y estas amenazas o riesgos que corre un individuo pueden convertirse en un ciberdelito. Por ejemplo, Álvarez (2009) afirma que el Internet tiene a lo que el autor llama su "reverso tenebroso", Ileno de intrusos, virus, timos, mafias, piratería, espionaje y pederastia. Por su parte, Burbules y Callister (2000), explican que en el Internet se puede pasar por experiencias o encuentros perturbadores o perjudiciales. Hay hostigamientos, amenazas, insultos, oferta de cosas no deseadas, pornografía, relatos de violencia, etc.; ellos lo llaman "un microcosmos de todo lo bueno y malo de la sociedad". Finalmente, los estudios de Livingstone (2007) sostienen tres tipos de riesgos principales: a) Los riesgos de contacto: surgen de la comunicación interpersonal a través de las TIC. Los jóvenes mantienen diálogos con amigos y compañeros a través de herramientas como el Messenger y diversas redes sociales, pero también cabe la comunicación con personas anónimas para ellos; b) Los riesgos de privacidad: estos hacen referencia de forma concreta a la invasión de la intimidad de una persona; y c). Los riesgos comerciales: son aquellos derivados de la actividad publicitaria y mercantil que se da a través de la Red.

\section{DisEÑO METOdOLÓGICO}

El estudio se planificó como una investigación de tipo descriptiva y para su implementación requirió de un enfoque mixto integrado que permite el análisis de datos de manera transversal y temporal, en este caso entre los años 2012, 2013 y 2014. Contempla dos fases metodológicas y una combinación de técnicas cuantitativas y cualitativas. El análisis cuantitativo contempló la aplicación de 2.907 cuestionarios aplicados a estudiantes de los tres grados de secundarias que fueron seleccionados en función de la representatividad de un universo de 27.379 alumnos. La muestra seleccionada la componen exclusivamente jóvenes estudiantes de secundaria del Municipio de Hermosillo, Sonora. Dicha muestra ha sido seleccionada en función de la 
representatividad de este universo y teniendo en cuenta variables como su distribución por sexo, edad y tipología del centro educativo. Para determinar la muestra representativa se utilizaron los siguientes parámetros: Error máximo aceptable: 2\%; Porcentaje estimado de la muestra: 99\%; Nivel deseado de confianza: 99\%; Tamaño del universo: 27.379 alumnos; La muestra final encuestada: 2.907 casos aplicados. El cuestionario se aplicó a la totalidad de centros escolares públicos del Municipio de Hermosillo, Sonora; México.

La fase cualitativa del diseño de investigación fue realizada con la implementación de la técnica de grupo focal. Se organizó de la siguiente manera: a partir del universo y muestras de las secundarias aplicadas en el estudio cuantitativo y de los datos proporcionados por el portal del Instituto de Evaluación Educativa del Estado de Sonora, se seleccionaron diversas secundarias públicas del Municipio de Hermosillo, Sonora; México. La selección se realizó exclusivamente a partir de las condiciones favorables y desfavorables (desde el punto de vista técnico y humano de participación y colaboración que presentaban) para realizar la implementación del grupo focal. A partir de aquí, cada centro escolar determinó el día, el lugar y el personal de orientación y apoyo del plantel que colaboraría con el grupo de investigación para realizar la aplicación del grupo focal. Posteriormente, con apoyo del mismo personal de orientación vocacional de cada centro, se eligieron alumnos de los tres grados, ambos sexos. El muestreo final realizado fue de tipo intencional a partir de la selección previa de sujetos tipo con base en determinados criterios de inclusión donde se destacan la conexión a Internet (accesibilidad) y la utilización frecuente de algún dispositivo móvil con Internet. En cada grupo focal se contó con personal de apoyo como apuntadores y apoyo técnico de equipo audiovisual. El o la moderadora siempre fue un miembro responsable del grupo de investigación. Entre los recursos técnicos utilizados se encuentran la grabadora de voz digital, la cámara de fotos digital y la cámara de audiovisual. Los sujetos que participaron en los siete grupos focales aplicados. Recordemos que, desde la óptica cualitativa, el muestreo es de tipo intencional y se configura a partir de la selección previa de sujetos tipo con base en determinados criterios de inclusión. En la metodología cualitativa no es apropiado tratar de cumplir con los cánones de validez y fiabilidad. La mayoría de las investigaciones en esta perspectiva han trabajado para desarrollar otros criterios para evaluar la calidad de los datos cualitativos. Estos están relacionados, en su mayor parte, con las opiniones de los individuos que la llevan a cabo. Por ejemplo, Guba y Lincoln (1989), han introducido una serie de normas de validación de alternativas dentro de la tradición interpretativa que son análogos a los conceptos convencionales de validez y fiabilidad. Por nuestra parte hemos tratado de enfatizar como criterio principal los propios resultados que se venían generando en los distintos análisis de datos que se generaban de manera transversal y temporal en la investigación. Así, nos centramos en definir cómo las interpretaciones se basan en 
los datos y si se formulan, en todo caso, de manera consistente con los datos cualitativos y cuantitativos disponibles con anterioridad.

\section{RESULTADOS DEL ESTUDIO}

Con base en el análisis de los resultados obtenidos provenientes de los instrumentos cuantitativo y cualitativos y partiendo de las conclusiones preliminares arrojadas por los sujetos de estudio (alumnos), en respuesta a los apartados de uso, socialización, regulación y control de padres y profesores, se generan a continuación los siguientes hallazgos generales:

\subsection{Usos, consumos y preferencias en Internet}

La variable asociada del uso de Internet está fuertemente asentado en la vida cotidiana de los jóvenes inscritos en secundarias públicas: el 93\% de los jóvenes entre 12 y 15 años usa Internet al menos una vez a la semana. El 60\% utilizan Internet diariamente entre dos y cinco horas. Los menores acceden a Internet cada vez más jóvenes. El lugar más común para el uso de Internet es el hogar, seguido de la escuela. Sin embargo, el acceso a Internet se está diversificando, en su dormitorio y a través de su teléfono móvil u otro dispositivo portátil. Los jóvenes desarrollan una diversa gama de actividades potencialmente beneficiosas online. Por ejemplo, habilidades digitales. En el caso mexicano, es altamente probable que un mayor uso contribuya a la alfabetización digital y las habilidades relativas a la seguridad. Sus actividades se vuelven multitareas y multifuncionales además son creadores de contenidos. Además, la mayoría de ellos, aprendieron a utilizar la TIC de manera autodidacta; lo cual puede ser un indicador para desarrollar futuros programas que incluyan esta habilidad. En cuanto a las preferencias, es marcada la tendencia hacia el uso para ocio y entretenimiento, sumado a esto, el tiempo que cada joven dedica al día a estar conectado, muestra un uso excesivo de las TIC en comparación al tiempo dedicado a actividades escolares. Sin embargo, este hecho no es relevante para el joven, ya que ellos consideran que no le han quitado tiempo a ninguna otra actividad, incluyendo las actividades escolares. Sobresale la predilección por las redes sociales, videojuegos, juegos en línea y contenidos de entretenimiento de video y música. Los nativos digitales disfrutan de una posición multi-canal: pueden prestar atención a muchas cosas. Simultáneamente, juegan en la Web, chatean, intercambian SMS, charlan con los que están con ellos y hablan por el celular. 


\subsection{Socialización. Interacción comunicativa}

Otra de las variables centrales del estudio es la socialización en las relaciones interactivas en los jóvenes de secundaria. De acuerdo con los resultados arrojados, el $56,6 \%$ de los estudiantes de secundaria si tienen amigos virtuales, y el $43,4 \%$ de los jóvenes señaló que no tienen amigos virtuales. Ve manera muy similar que los estudiantes de secundaria opinaron de manera muy semejante a dos años de haber realizado el segundo levantamiento de información, en comparación con en el estudio anterior cuando se les cuestionó si tienen amigos virtuales. Tal y como ha sucedido en estudios previos, se reafirma que los procesos de interacción a través de Internet no están en declive como se argumentará más adelante. En correspondencia con levantamientos anteriores, se pudo comprobar que los jóvenes manifestaron con un 56,6\% tener amigos virtuales. Los jóvenes mantienen contacto permanente con sus amigos, conocen personas con las que comparten intereses y aficiones, y amplían conocimientos y habilidades. Internet estaría permitiendo a los jóvenes extender y fortalecer sus relaciones sociales. Con respecto a esta variable asociada se puede concluir que si bien el proceso de socialización es sano para la construcción de las identidades, tal y como se señalan en otros estudios al compartir intereses particulares, debemos hacer hincapié de que existe un gran riesgo en la socialización interactiva al darse un alto índice de estudiantes que afirma tener amigos virtuales, sin considerar en ocasiones, que no sabemos quién o quiénes están del otro lado de la pantalla.

\subsection{Riesgo. Regulación, mediación y control de padres y profesores}

En la medida en que el uso de Internet se ha vuelto cada vez más personal, el rol de padres y profesores es cada vez más difícil. Esto implica una gran responsabilidad por parte de tutores y las propias industrias prestadoras de servicio de Internet para gestionar la prevención de riesgos que los menores pueden encontrar. Es también una oportunidad para proporcionar herramientas que eviten o superen dichos riesgos. Esto también supone una mayor responsabilidad sobre su propia seguridad, por lo que los mensajes sobre la seguridad en Internet tienen que dirigirse a aumentar la confianza, la resistencia y las competencias digitales entre los menores.

Desde el seno familiar y el centro escolar, la formación en las habilidades digitales es necesaria para asegurar que todos los menores alcancen unos conocimientos mínimos para prevenir que algunos menores pueden quedarse aislados sin capacidad de afrontar los riesgos que les puedan aparecer. Lo anterior igualmente implica tratar de ampliar el abanico de actividades que desarrollan los menores en Internet, ya que son muy pocos los que desarrollan actividades creativas. Esta generación interactiva con un sólo vistazo pueden intuir cuáles son los botones apropiados para hacer que cualquier aparato funcione, a la vez pierden conciencia de los procedimientos que son 
necesarios para producir un resultado. Esto hace que se desesperen ante aquello que demanda concentración o que requiere de procesos lentos y esforzados para dar resultados.

\section{CONCLUSIONES. VARIABLES ASOCIADAS}

Una primera variable asociada identificada en el presente estudio es el "sentido del apego y preferencia" en el uso y el consumo del dispositivo portátil inteligente con conectividad. Se ha identificado que el principal atractivo que los jóvenes encuentran en la red es la posibilidad de socializar, compartir información y comunicarse. Sin embargo, el uso educativo o académico no figura entre sus principales usos y consumos. En nuestra opinión ello representa uno de los principales desafíos para los responsables de la educación formal en los espacios educativos, en este caso, en la educación básica del nivel secundaria en México. Los planes y programas de estudio de la secundaria pública mexicana están enfocados en el desarrollo de habilidades digitales. Sin embargo, definitivamente no concuerdan con la realidad en las preferencias de los jóvenes al utilizar Internet. Destaca notablemente el uso de las TIC en los jóvenes es el uso para el ocio y entretenimiento. Es en este indicador donde despunta la participación de los menores. En su gran mayoría son ellos mismos los que se consideran casi expertos en el uso de software y aplicaciones que les permiten crear contenidos, editar imágenes, música, video y fotografía. Reconocen, identifican y utilizan gran variedad de sitios en los cuales pueden publicar, compartir, comentar y participar de manera activa. La variable asociada de "sentido del apego y preferencia" hacia las redes sociales se caracteriza por encontrar contenidos entretenidos que los mantengan motivados. Se materializa al ver y oír videos, música, series e interactuar con juegos (preferentemente) en línea.

Otra variable asociada con la socialización y que en nuestro estudio juega un papel relevante es lo que aquí denominamos como "socialización en pares en línea". Se identifica mediante las conversaciones en vivo y concentran una alta posición de atención e interactividad sobre todo cuando la comunicación y la socialización se realizan de manera anónima. La mayoría de los sitios donde cobra vida la "socialización en pares en línea" instrumenta algún filtro de restricción, sobre todo la ligada con la edad del participante, pero al ser públicos y de acceso abierto, no existe la manera de comprobar el estatus ni la identificación puntual del mismo. Este tipo de actividad vinculada a dicha variable asociada es una de las prácticas más riesgosas, ya que cualquier individuo puede interactuar con los menores, por ejemplo. Una preocupación presente en las interacciones comunicativas es la identidad de la gente con la que interactúan. Se ha dado mayor importancia al equipamiento de tecnología que a los propios contenidos y sujetos participantes. Es ahí donde radica la principal razón 
de salvaguardar la seguridad de los menores. En tanto la investigación cualitativa permitió corroborar y profundizar aún más en la comprensión de cómo es que se construyen las identidades de los jóvenes de secundaria, a través de la "socialización en pares en línea". Para los adolescentes el estar comunicado por las redes sociales es primordial para la construcción de sus identidades. Los testimonios de éstos, destacaban la presencia de la confrontación con los grupos, cuando algo no les parecía bien lo manifestaban a través de las redes sociales, alterando, en ocasiones, los ánimos de quienes ven ciertas publicaciones por no compartir o estar de acuerdo con las ideologías de quienes expresaban su sentir. En nuestra opinión se trata de un uso evidente, primordial en esta fase del ciclo vital de su vida, ya que el individuo utiliza la interacción y la comunicación con otros como forma de gestionar su estatus y consolidar los roles determinados entre los miembros de la comunidad con la que convive. De hecho, un factor de exclusión social deviene de la "no posesión de un dispositivo inteligente (ejemplo el móvil o celular inteligente), ya que esto los deja fuera de la comunidad inter-pares. Internet es el medio que le permite a la juventud actual el extender su red de relaciones sociales, conocer amistades y compartir con las ya existentes su rutina diaria y sus intereses. Por tanto, no es difícil entender que las redes sociales, como espacios de autoexpresión, conexión y construcción de relaciones interactivas, se han convertido en entornos fundamentales para la producción y la consolidación de la identidad en las y los adolescentes.

En el periodo de 2012-2014 se observó que la mayoría de los escolares si tiene amigos virtuales. Sin embargo, podemos concluir que las formas de sociabilidad en los jóvenes y las diversas formas de interacción se construyen a partir de sus relaciones en Internet. Un considerable porcentaje de estudiantes manifestó que Internet le facilita la comunicación, por lo cual le es muy útil e indispensable. De la misma manera, expresaron que les ayuda a ahorrar tiempo. Se encontró que algunas prácticas propias de los jóvenes en el espacio virtual consisten en subir fotografías o escribir diarios; además, una práctica bastante frecuente se define en el hecho de incorporar procesos de auto-revelación íntima del adolecente. Lo anterior nos ha expuesto la existencia de diversas formas de negociar el estatus de identidad del joven. También, que el joven genere oportunidades para la comparación social y la posibilidad de expresar aspectos idealizados virtualmente de quien se pretende ser o se querría ser. Los perfiles virtuales pueden entenderse como un cuerpo digital en el que los individuos se encarnan al escribir sobre sí mismos.

Además, queda también claro que en el caso de las redes sociales también sirven para medir las opiniones y la consideración que los demás tienen de uno mismo o de nuestros pares cercanos o grupos de amigos(as) cercanos. Por tanto, consideramos que definitivamente no es difícil entender que las redes sociales, como espacios de autoexpresión, conexión y construcción de relaciones, se han convertido en entornos 
fundamentales para la producción y la consolidación de la identidad en las y los adolescentes. Por último, dentro de los hallazgos cualitativos, habría que enfatizar el hallazgo de la variable asociada con la identidad cultural. Se puede concluir, mediante los hallazgos presentados que las y los adolescentes de secundaria comparten rasgos de estilos de vida así como una misma ideología en los diferentes ámbitos de una sociedad. Principalmente en el nivel personal del joven adolescente pero también de manera colectiva.

Finalmente, la portabilidad y accesibilidad de los dispositivos ha acelerado la adquisición de una última variable asociada identificada en este estudio: lo que llamamos el "patrón de conducta antepuesta en el individuo". Es decir, el uso excesivo o dependencia excesiva del dispositivo inteligente cuyos extremos llegan a una posesión de 24 horas diarias y cuyas repercusiones están vinculadas directamente con su desempeño escolar, con la salud física y mental del adolecente, entre otros efectos negativos descubiertos. Puntualmente nos referimos aquí a la conducta y al estado emocional del joven que aparece como reacción de adaptación o respuesta a situaciones cambiantes y diversas de su vida como adolecente. De acuerdo con los datos del estudio cualitativo, la justificación de la variable asociada que llamamos "patrón de conducta antepuesta en el individuo" está vinculada con un contexto de surgimiento basado en "la manifestación de aburrimiento constante en su vida diaria", ya que al no tener "nada que hacer" es fácil recurrir a distraerse o entretenerse en las redes sociales. Hemos podido documentar, sobre todo a partir del trabajo con grupos focales, que el joven desarrolla niveles de ansiedad considerados por el mismo como algo "normal" porque fundamentalmente considera que el estar conectado mediante algún tipo de dispositivo inteligente les "estimula" y les "beneficia" para "entablar nuevas relaciones en su actividad cotidiana como adolecentes" o porque "simplemente entretiene".

Lo que el joven no deja claro, aunque se acepte como una conducta generalizada, es habitualizar la conducta de la "conectividad permanente" y que definitivamente le impide realizar determinadas actividades que antes realizaba como, por ejemplo, socializar directamente -cara a cara- con familiares y amigos. En definitiva ello lleva al joven a realizar diversos comportamientos y le conduce también a enfermedades orgánicas o psicológicas que no le son de su agrado. Se debe también señalar que en algún momento, si queda claro para el joven que su preferencia podría dirigirse a realizar alguna otra actividad como salir con amigos o alguna otra actividad física o deportiva. Incluso, algunos menores afirmaron que las restricciones hechas por sus padres y profesores en cuanto a limitar en tiempo de uso, en ocasiones lo consideran necesario y que no les molesta si los limitaran ocasionalmente. 
Consideramos que es en los padres en quién recae la responsabilidad de regular el uso de Internet a través de cualquier dispositivo. Son los padres los principales proveedores de tecnología a sus hijos. De acuerdo con la evidencia encontrada, parece quedar claro que entre mayor dominio tengan padres y profesores en el uso de los dispositivos inteligentes $y$, en general, de la tecnología digital, mayor será el conocimiento y el sentido de prevención de riesgo para diseñar y aplicar estrategias de regulación en el uso seguro de Internet.

Concluimos que son las instituciones escolares las que juegan (o deberían jugar) un papel fundamental para brindar oportunidades de alfabetización y actualización permanente tanto a padres como a maestros. Incorporar las TIC al aula es una necesidad urgente. Antes que se menoscaben las habilidades de estas nuevas generaciones, es necesario nivelar la balanza. Aprovechar que los jóvenes tienen el conocimiento del uso de las tecnologías. El reto será trasladar esos conocimientos al ámbito académico y que los jóvenes sean capaces de crear contenidos educativos que nutran y aporten a su acervo académico y realmente desarrollen las competencias que los modelos educativos y la sociedad en general exige de ellos.

Por último queremos también enfatizar el hecho que entre el periodo 2012-2014 se presentó un incremento del $40 \%$ la navegación en solitario. Es decir, los menores de edad se encuentran menos vigilados, con mayor acceso (tiempo y conectividad) y mayormente expuestos. No es casualidad que en correspondencia se hayan incrementado los casos de difamación, acoso, suplantación de identidad y ciberbullying, entre otras conductas tipificadas como delito de acuerdo con el código federal de procedimientos penales vigente en México. En el estudio ha quedado claro que la mediación y la regulación que realizan los padres frente al Internet se focaliza en la orientación de no contactar a personas desconocidas por medio de las redes sociales. La privacidad es un factor determinante pero sólo en algunas circunstancias en particular. Es importante mantener la privacidad con respecto a sus padres. También consideran importante mantener en bajo perfil su propia identidad e incluso sus propios datos personales como dirección y nombre, no así su propia imagen, incluyendo aquellas fotografías expuestas en circunstancias por demás inapropiadas (desnudas o semidesnudas, ingiriendo alcohol, etc.). De hecho, por lo general el joven considera poco relevante cuando ha sido víctima de difamación ya sea por comentarios en texto y/o imagen difundida en alguna red social. Lo que se destaca por parte de los jóvenes, es la indiferencia ante los frecuentes casos de fotografías publicadas con imágenes de menores desnudas o semidesnudas. Los mismos alumnos comentaron que son los más jóvenes (de primer grado se secundaria) los que acostumbran a realizar esta práctica. En la información generada a partir de los grupos focales se mencionan varios casos de niñas de sexto de primaria. Todo parece indicar que es ésta una práctica frecuente entre el género femenino. Son ellas las que generalmente tienen la ini- 
ciativa de auto-fotografiarse y compartir las imágenes con amigas o novios. En algún momento de este proceso de socialización y de comunicación actual se quebranta la confianza y un "tercero" comparte las imágenes, mismas que se difunden de manera viral en la red. La totalidad de las instituciones educativas visitadas presentaron casos de este tipo. Ninguna cuenta con un plan o estrategia para resolver estas situaciones. Las trabajadoras sociales de los centros escolares visitados y a las cuales se entrevistó comentaron que ante estas situaciones los padres de familia toman una postura radical ante la defensa de sus hijos, negando toda posibilidad de participación intencional del afectado. Profesores, directivos y trabajadores sociales del centro escolar han tenido que afrontar conflictos entre padres tratando de buscar una solución y en casos particulares han intervenido autoridades educativas y judiciales. Incluso, en algunos casos, se han solucionado desde instancias penales donde se trata a las y los jóvenes como infractores menores con sus respectivas sanciones y sentencias penales.

\section{BibLIOGRAFÍA}

ÁlVAREZ, G. (2009): Cómo protegernos de los peligros de Internet. Madrid: Consejo Superior de Investigaciones Científicas.

BERGER, P. y LUCKMANN, T. (1968): La construcción social de la realidad. Buenos Aires: Amorrortu.

BERGER, P. y LUCKMANN, T. (1997): Modernidad, pluralismo y crisis de sentido. Barcelona: Paidós.

BOWMAN, L., WAITE, B. y LEVINE, L. (2014): “A cross-cultural comparison of media multitasking in American and Malaysian college students", International Journal of Cyber Behavior, Psychology and Learning, vol. 4, no 3, pp. 1-16. DOI: http://dx.doi.org/10.4018/ijcbpl.2014070101.

BRINGUÉ, X., SÁDABA, C. y RODRÍGUEZ, J. (2008): La Generación Interactiva frente a un nuevo escenario de comunicación: Retos sociales y educativos. Navarra: Consejo Audiovisual de Navarra.

BUCKINGHAM, D. (2013): Media education: Literacy, learning and contemporary culture. John Wiley \& Sons.

BURBULES, N. y CALLISTER, T. (2000): Watch it: The risks and promises of information technologies for education. Boulder, Colorado: Westview.

CRAIG, R. (1999): "Communication theory as a field", Communication Theory, vol. 9, no 2, pp. 119-161. DOI: http://dx.doi.org/10.1111/j.1468-2885.1999.tb00355.x. 
CRAIG, R. y TRACY, K. (2014): "Building grounded practical theory in applied communication research: Introduction to the special issue", Journal of Applied Communication Research, vol. 42, no 3, pp. 229-243. DOI: http://dx.doi.org/10.1080/00909882.2014.916410.

DÍAZ BARRIGA, Á. (2005): Integración a las TIC en el currículo y la enseñanza para promover la calidad y la innovación. Ciudad de México: UNAM.

DUERAGER, A. y LIVINGSTONE, S. (2012): How can parents support children's internet safety? London: EU Kids Online.

EYNON, R. y HELSPER, E. (2014): "Family dynamics and Internet use in Britain: What role do children play in adults' engagement with the Internet?", Information, Communication \& Society, vol. 18, no 2, pp. 156-171. DOI: http://dx.doi.org/10.1080/1369118X.2014.942344.

GARCÍA, F. (2010): Internet en la Vida de Nuestros Hijos ¿Cómo transformar los riesgos en oportunidades? Pamplona: Foro Generaciones Interactivas.

GARITAONANDIA, C. (2006): Oportunidades y riesgos para los niños y jóvenes en Internet. London: Staksrud.

GUBA, E. (1989): Fourth Generation Evaluation. Newbury Park: Sage.

HANSSON, B. (1999): "Interdisciplinarity: For what purpose?”, Policy Sciences, vol. 32, no 4, pp. 339-342. DOI: http://dx.doi.org/10.1023/A:1004718320735.

HUERTAS, A. y FIGUERAS, M. (2014): Audiencias juveniles y cultura digital. Barcelona: Instituto de la Comunicación, Universidad Autónoma de Barcelona.

LENHART, A. y MADDEN, M. (2007): Teens, Privacy \& Online Social Networks. How teens manage their online identities and personal information in the age of MySpace. Washington: Pew Internet \& American Life Project.

LENHART, A., MADDEN, M. y SMITH, A. (2008): Teens and Social Media: The use of social media gains a greater foothold in teen life as them embrace the conversational nature of interactive online media. Washington: Pew Internet \& American Life Project. Disponible en http://www.pewinternet.org. Consultado el 23 de mayo de 2014.

LENHART, A, PURCELL, K, SMITH, A. y ZICKUHR, K. (2010): Social media and mobile Internet use among teens and young adults. Washington: Pew Internet \& American Life Project. Disponible en http://www.pewinternet.org/Reports/2010/Social-Media-and-Young-Adults.aspx. Consultado el 19 de abril de 2014.

LIVINGSTONE, S. (2002): Young people and new media: Childhood and the changing media environment. London: Sage.

LIVINGSTONE, S. (2007): "Do the media harm children? Reflections on new approaches to an old problem", Journal of Children and Media, vol. 1, no 1, pp. 5-14. DOI: http://dx.doi.org/10.1080/17482790601005009. 
LIVINGSTONE, S. (2008): "Taking risky opportunities in youthful content creation: teenagers' use of social networking sites for intimacy, privacy and self-expression", New Media \& Society, vol. 10, $n$ o 3, pp. 393-411. DOI: http://dx.doi.org/10.1177/1461444808089415.

LIVINGSTONE, S. (2010): “On the rapid rise of social networking sites: New findings and policy implications", Children \& Society, vol. 24, no 1, pp. 75-83. DOI: http://dx.doi.org/10.1111/j.1099-0860.2009.00243.x.

LIVINGSTONE, S. (2014): “¿In their own words: What bothers children online?”, European Journal of Communication, vol. 29, no 3, pp. 271-288. DOI: http://dx.doi.org/10.1177/0267323114521045.

LEÓN DUARTE, G. A. (2014): Comunicación y Sociedad en América Latina. Publicia.

LEÓN DUARTE, G. A. y ABRIL VALDEZ, J. A. (2013): “Interactive digital communication and new analysis approaches in cultural practises: the interdisciplinary perspective on the study of audiovisual production", Revista Mediterránea de Comunicación, vol. 4, № 1, pp. 85-98. DOI: http://dx.doi.org/10.14198/MEDCOM2013.4.1.04.

LEÓN, G., CASTILLO, E. MONTES, M. y CAUDILLO, D. (2013): Relaciones interactivas, Internet y jóvenes de secundaria en México. Primera oleada sobre usos, consumos, competencias, y navegación segura de Internet en Sonora (2012). Hermosillo: SEP-Gobierno del Estado de Sonora-Qartuppi-Universidad de Sonora.

LEÓN, G., CAUDILLO, D., CONTRERAS, C. y MORENO, D. (2014): Jóvenes y medios digitales móviles en México. Un estudio de variables asociadas en perspectiva interdisciplinar. Hermosillo: SEP-Universidad de Sonora.

MORIN, E. (1998): "Sobre la Interdisciplinariedad", Boletín n² 2 del Centre International de Recherches et Etudes Transdisciplinaires.

UNESCO (2010): Informe sobre las Ciencias Sociales en el mundo. Las brechas del conocimiento. Paris: UNESCO.

PATRY, J.-L. (2013): "Beyond multiple methods: Critical multiplism on all levels", International Journal of Multiple Research Approaches, vol. 7, ํo 1, pp. 50-65. DOI: http://dx.doi.org/10.5172/mra.2013.7.1.50.

PURCEL, K. HEAPS, A., BUCHANAN, J. y FRIEDRICH, L. (2013): How teachers are using technology at home and in their classrooms. Washington: Pew Research Center's Internet \& American Life Project.

REPKO, A. (2008): Interdisciplinary Research. Process and Theory. Sage: California.

SHADISH, W. (1986a): "The person-situation debate: A critical multiplist perspective", Journal of Personality, vol. 54, no 1, pp. 52-105. DOI: http://dx.doi.org/10.1111/j.14676494.1986.tb00390.x.

SHADISH, W. (1986b): "Planned critical multiplism: Some elaborations", Behavioral Assessment, 8, pp. 75-103. 
SHADISH, W. (1993): "Critical multiplism: A research strategy and its attendant tactics", New Directions for Program Evaluation, no 60, pp. 13-57. DOI: http://dx.doi.org/10.1002/ev.1660.

SILBEREISEN, R., RITCHIE, P. y OVERMIER, B. (2010): "La psicología en el vórtice de la convergencia y la divergencia: el caso del cambio social", en VV.AA.: Informe mundial sobre las ciencias sociales. México: UNESCO / Foro Consultivo 2011, pp. 222-226.

STAKSRUD, E., ÓLAFSSON, K. y LIVINGSTONE, S. (2013): "Does the use of social networking sites increase children's risk of harm?", Computers in Human Behavior, vol. 29, no 1, pp. 40-50. DOI: http://dx.doi.org/10.1016/j.chb.2012.05.026.

VALKENBURG, P., PETER, J. y SCHOUTEN, A. (2006): "Friend networking sites and their relationship to adolescents' well-being and social self-esteem", CyberPsychology \& Behavior, vol. 9, no 5, pp. 584-590. DOI: http://dx.doi.org/10.1089/cpb.2006.9.584.

Gustavo Adolfo LEÓN DUARTE es líder del Grupo Enseñanza e Investigación de la Comunicación en América Latina (GEIC-AL), Coordinador del Posgrado Integral en Ciencias Sociales de la Universidad de Sonora, Licenciado en Ciencias de la Comunicación por la Universidad de Sonora y Master y Doctor en Periodismo y Ciencias de la Comunicación por la Universidad Autónoma de Barcelona, España. Es Miembro del Sistema Nacional de Investigadores de México (Consejo Nacional de Ciencia y Tecnología de México - CONACYT, México). Ha impartido cátedra y conferencias en pre y postgrado en varias universidades de EE.UU. e Hispanoamérica. Ha publicado 15 libros y más de 80 artículos en libros y revistas especializadas. Sus libros más recientes son Estudios de la Comunicación. Estrategias Metodológicas y Competencias Profesionales en Comunicación (Pearson-Prentice Hall: 2012); Relaciones Interactivas, Internet y Jóvenes de Secundaria en México (SEP-QartuppiUniversidad de Sonora, 2013) y Jóvenes e Internet Seguro en México (SEP-Qartuppi-Universidad de Sonora, 2014). Correo electrónico: gustavoadolfoleon@gmail.com.

Dora Yéssica CAUDiLlo RuIz es estudiante de Doctorado en el Posgrado Integral en Ciencias Sociales de la Universidad de Sonora. Es Licenciada en Ciencias de la Comunicación por la Universidad de Sonora. Maestría en Educación por la Universidad Pedagógica Nacional. Es Miembro del Grupo Enseñanza e Investigación de la Comunicación en América Latina (GEIC-AL). Ha publicado varios artículos y capítulos de libro. Correo electrónico: doracaudilloruiz@gmail.com.

Carlos René Contreras CÁZARez es miembro del Grupo de Enseñanza e Investigación de la Comunicación en América Latina (GEIC-AL). Es Licenciado en Ciencias de la Comunicación por la Universidad de Sonora. Es Máster en Periodismo Avanzado en Investigación por la Universidad Ramón Llull y Maestro en Ciencias Sociales por el Posgrado Integral en Ciencias Sociales (PICS) de la Universidad de Sonora, México. Ha publicado varios artículos y capítulos de libro. Correo electrónico: renecazarez@gmail.com. 